\title{
The Scenario of Dengue-like Illness in Paediatric Population of Southern Bangladesh
}

\author{
M Luthfor Rahman, ${ }^{1}$ AKM Mamunur Rashid, ${ }^{2}$ Mithun Debnath, ${ }^{3}$ Tasnuva Islam ${ }^{4}$
}

\begin{abstract}
Dengue is one of the most important mosquito-borne viral diseases affecting humans. Dengue infections can result in a broad spectrum of disease severity ranging from an influenza-like illness (DF) to the life-threatening dengue hemorrhagic fever (DHF)/dengue shock syndrome (DSS). Although adults do experience shock, vascular leakage is generally more severe in young children, and in endemic areas, DSS is seen primarily in the pediatric population. The detailed clinical and laboratory profile is crucial for the diagnosis and the successful management of the patients.

This study aims to observe the clinical features and laboratory results for the better understanding of patient management to reduce child death in case of dengue fever. A prospective cross-sectional study was conducted at the dengue ward of Khulna Medical College Hospital; 167 suspected dengue patients were enrolled in our research during the period from 7th August to 8th November 2019. Patients attending the emergency department and pediatric outpatient department with complaints of fever and features of dengue with positive NS1 antigen test and/or positive dengue specific antibody $\lg M$ or $\lg G$ or both were included in this study. Diagnosis of dengue was made on the basis of compatible clinical features and NS1 antigen positivity and/or detection of IgM and IgG antibodies. Patients were classified according to WHO 2012 protocol, and data was analyzed.
\end{abstract}

One hundred sixty patients $(95.8 \%)$ were diagnosed as having dengue illness, and the rest $7(4.2 \%)$ as fever other than dengue. Among the dengue illness, dengue fever (DF) $87(52.1 \%)$, DF with warning signs $51(30.5 \%)$, DHF $19(11.4 \%)$, and DSS $3(1.8 \%)$. The most common signsymptoms of dengue was fever(100.0\%),vomiting( $52.1 \%)$,abdominal pain( $38.3 \%)$, headache(23.4\%),skin rash(13.25\%), melena $(7.8 \%)$, abdominal tenderness $(28.7 \%)$,palpable liver (24.6\%), and palpable spleen(3.0\%). NS1 antigen was found positive in $88.6 \%$ cases, negative $3.0 \%$, and NS1 antigen test not done $8.4 \%$ cases due to time constrain. Anti-dengue antibody IgM positive in $10(6.0 \%)$, Ig G positive $7(4.2 \%)$,both IgM \& IgG positive $7(4.2 \%)$,both IgM \& IgG negative $57(34.1 \%)$ and antibody test not done in $86(51.5 \%)$ cases due to time constrain. Antidengue antibody not done in $86(51.5 \%)$ cases; among them most of the cases $(98.8 \%)$ are found NS1 positive and $1.2 \%$ NS1 test negative $(P<0.001)$ in our study which is statistically significant.

Observation of clinical and laboratory parameters is helpful for dengue classification and management to reduce child death.

Key words: Dengue, clinical features, NS1.

TAJ 2021; 34: No-1: 86-96

${ }_{1}^{1}$ Assistant Professor (Paediatrics) OSD, DGHS, Mohakhali, Dhaka, Attached to Naogaon Medical College.

2 Head, Department of Pediatrics, Khulna Medical College.

${ }^{3}$ Assistant Registrar (Pediatrics) Khulna Medical College Hospital.

${ }^{4}$ Assistant Registrar (Peediatrics) Khulna Medical College Hospital. 


\section{Introduction}

Dengue is one of the most important mosquitoborne viral diseases affecting humans. Infection can be caused by any one of four dengue viruses (DENV-1 to DENV4), transmitted by Aedes mosquitoes. ${ }^{1}$ These four serotypes are genetically similar and share approximately $65.0 \%$ of their genomes. The fifth variant DEN-5 has been isolated in October $2013 .^{2}$ Infection with one of the serotypes confers serotype-specific lifelong immunity; however, secondary infection with a heterogenous serotype often creates devastating outcomes, which may be due to antibodydependent enhancement. ${ }^{3}$ These infections may be asymptomatic or may lead to (a) "classic" dengue fever(DF), or (b) Dengue hemorrhagic fever(DHF), or (c) Dengue hemorrhagic fever with shock, also known as Dengue shock Syndrome(DSS). ${ }^{4}$ An estimated 2.5 billion people worldwide are at risk of dengue. More than 50 million dengue infections are estimated to occur annually, of which approximately 500,000 result in hospital admissions for severe dengue in the form of dengue hemorrhagic fever (DHF) or dengue shock syndrome(DSS), principally among children. ${ }^{5}$ At present, dengue is endemic in 112 countries in the world ${ }^{6}$. Approximately $36 \%$ of annual dengue infections occur among children aged 1-9 years, and the average age of infection is 12 years. ${ }^{7}$ Ninety percent of DHF occur in children less than 15 years of age. ${ }^{8}$ The disease has a seasonal pattern, and the cases peak after the monsoons. ${ }^{9}$ The most notable complication of dengue is an unexplained vasculopathy that manifests as a transient increase in vascular permeability resulting in leakage of plasma from the circulation. Substantial plasma losses may occur, leading to the potentially fatal dengue shock syndrome (DSS). Although adults do experience shock, vascular leakage is generally more severe in young children ${ }^{9}$ and in endemic areas DSS is seen primarily in the paediatric population. There is currently no specific treatment for DENV infection $^{8}$ although several potential vaccines are in development; therefore, the only method of preventing transmission is vector control. ${ }^{6}$ In 2009 World Health Organization (WHO) revised the classification for dengue, defining two major entities dengue and severe dengue. Severe dengue is characterized by severe plasma leakage leading to shock, fluid accumulation with respiratory distress, severe bleeding and severe organ involvement evidenced by AST or ALT > 1000, impaired consciousness, cardiac and other organ involvement. ${ }^{10}$ The new classification also encompasses a set of "Warning signs" intended to help clinicians identify patients likely to develop complications during the critical phase of the illness. Dengue infections can result in a wide spectrum of disease severity ranging from an influenza-like illness (DF) to the life-threatening dengue hemorrhagic fever (DHF)/dengue shock syndrome $(\mathrm{DSS})^{8}$. The exact clinical and laboratory profile is crucial for diagnosis as well as for the successful management of the patients. The aim of this study is to observe the clinical features and laboratory results for a better understanding of patient management to reduce child death in case of dengue fever.

\section{Materials and Methods}

A descriptive type of cross-sectional study was conducted at the special dengue ward of Khulna Medical College Hospital over 167 suspected dengue patients during the period from $7^{\text {th }}$ August to $8^{\text {th }}$ November 2019. Patients who attended the emergency and pediatric outpatient department with complaints of fever and features of dengue with or without positive NS1 antigen test or dengue specific antibody $\operatorname{IgM}$ or $\operatorname{IgG}$ or both were included in the study. Diagnosis of dengue was made on the basis of compatible clinical features and NS1 antigen positivity and/or detection of $\operatorname{IgM}$ and $\operatorname{IgG}$ antibodies. NS1 antigen test was done in all patients with clinical features suggestive of dengue infection presenting within 5 days of onset of symptoms and patients who presented beyond 5 days of illness, denguespecific antibody IgM and IgG were done. A thorough physical examination was carried out in all cases. Data were collected by using a preformed questionnaire. The relevant investigations were done for every patient. According to WHO 2012 classification, patients were classified as dengue fever (DF), DF with 
warning signs, dengue hemorrhagic fever DHF), and DHF with shock, also known as dengue shock syndrome (DSS). Consent was taken from patients, parents, or caregivers. Data were analyzed using SPSS 16.0 program.

\section{Results}

A total of 167 patients were enrolled in this study. Among them, 99 (59.3\%) were boys, and 68 $(40.7 \%)$ were girls (Figure-I). The majority of the patients, 81(48.5\%), belonged to the age group 5 10 years, and the age distribution has been shown in (Table-I). In our study majority $(26.9 \%)$ of patients came from the Jessore district, followed by Khulna (20.4\%), Pirozpur (16.2\%), and Satkhira (13.2\%). Figure II showed the area of distribution of the patients. Traveling history was present at $4.8 \%$ cases, and history of the same type of illness at the family and at the neighbor was present $15(9.0 \%)$ and $17(10.2 \%)$ respectively (Table-II).The most common sign-symptoms of dengue was fever $(100.0 \%)$, vomiting $(52.1 \%)$, abdominal pain $(38.3 \%)$, headache $(23.4 \%)$ and melena $13(7.8 \%)$, abdominal tenderness $(28.7 \%)$, palpable liver (24.6\%), skin rash (13.2\%), palpable spleen $(3.0 \%)$ and tourniquet test was positive in $3.6 \%$ cases (Table-III \&Table-IV). It was found that in most of the cases $(92.8 \%)$ fever persisted for 7 days and $7.2 \%$ cases for more than 7 days
(Figure-III). About eighty (79.0\%) patients had temperatures less than $102^{0} \mathrm{~F}$ followed by $18.0 \%$ patients who had $102-104^{\circ} \mathrm{F}$ (Figure-IV). The studies revealed that diarrhea $(8.9 \%)$ and cough $(13.8 \%)$ were the commonly associated symptoms (Table-V). Leucopenia and thrombocytopenia were seen in $10.2 \%$ and $38.3 \%$ cases, respectively (Table-VI). Majority [85 (50.9\%)] patients had haematocrit value $30.0-35.0 \%$ and $31.1 \%$ patients had haematocrit value 35.0 - $45.0 \%$ (Table-VI). It was found that $88.6 \%$ of cases became NS1 antigen-positive, $3.0 \%$ of cases were negative (Table-VII). The study showed that $6.0 \%$ of patients became anti-dengue antibody IgM positive, $4.2 \%$ cases IgG positive, $4.2 \%$ had both IgM \& IgG positive, $34.1 \%$ cases became both IgM \& IgG negative, and antibody test not done in $86(51.5 \%)$ cases due to time constrain (TableVIII). Table IX shows anti-dengue antibody test not done in $86(51.5 \%)$ cases; among them, most of the cases $(98.8 \%)$ were NS1 positive and $1.2 \%$ NS1 negative $(P<0.001)$ in our study. One hundred sixty patients $(95.8 \%)$ were diagnosed as having dengue illness and the rest $7(4.2 \%)$ as fever other than dengue. Among the dengue illness; dengue fever (DF) 87 (52.1\%), DF with warning sign 51 (30.5\%), DHF $19(11.4 \%)$ and DSS $3(1.8 \%)$ [Table-X].

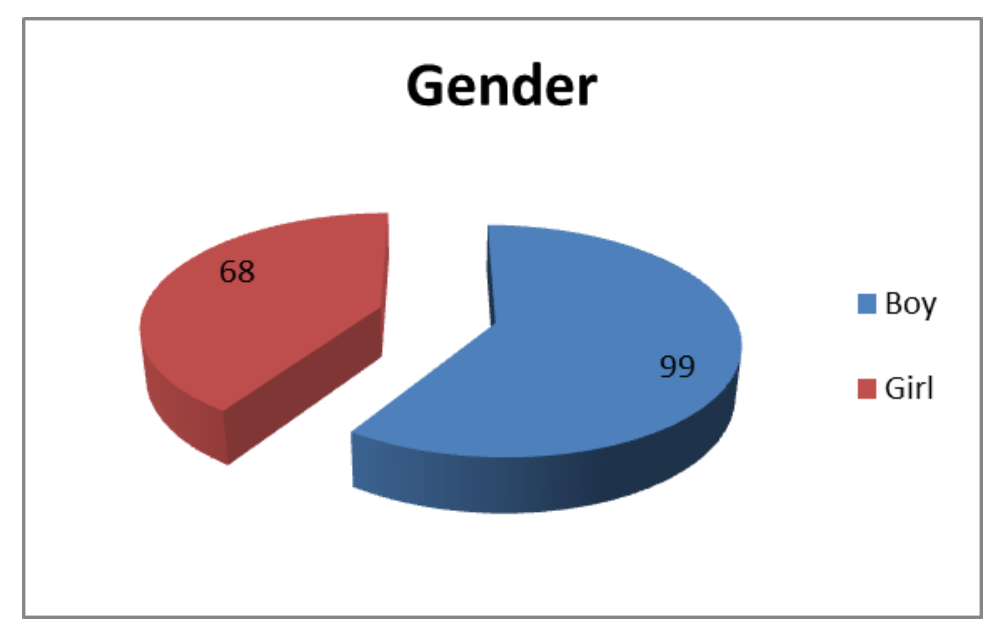

Figure I: Distribution of the respondents by gender 
Table I: Distribution of the respondents by age group

\section{Age Frequency Percent}

Less than 1year

10

6.0

$1-5$ years

51

30.5

5-10 years

81

48.5

Above 10 years

25

15.0

Total

167

100.0

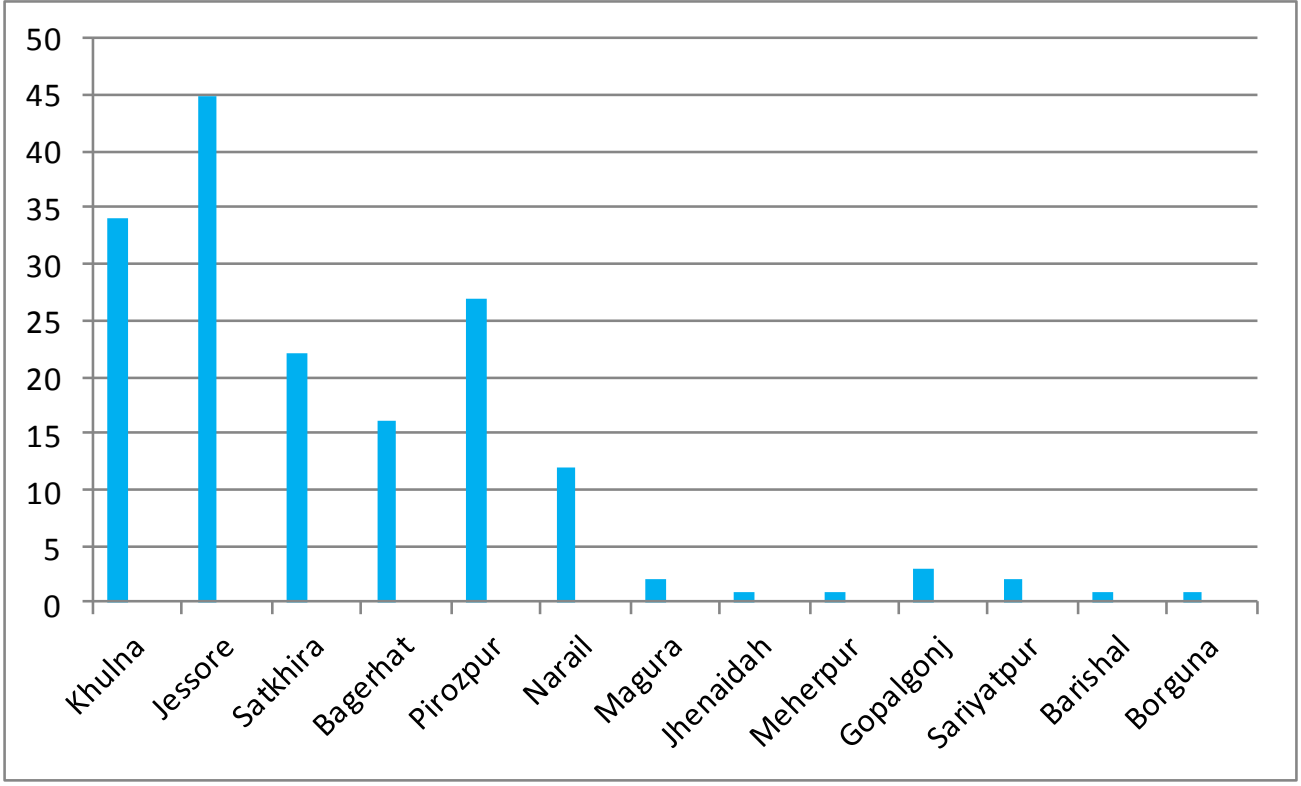

Figure II: Distribution of the respondents by area.

Table II: Distribution of the respondents by history

\section{History}

Frequency

8

159

15

152

17

150
Percentage

4.8

95.2

9.0

91.0

Same illness of neighbor

Yes

10.2

No 
Table III: Distribution of cases by symptoms

\begin{tabular}{lcc}
\multicolumn{1}{c}{ Symptoms } & Frequency & Percent \\
Fever & 167 & 100.0 \\
Headache & 39 & 23.4 \\
Retro-orbital pain & 8 & 4.8 \\
Bodyache & 40 & 24 \\
Joint pain & 9 & 5.4 \\
Vomiting & 87 & 52.1 \\
Abdominal pain & 64 & 38.3 \\
Convulsion & 2 & 1.2 \\
Skin rash & 22 & 13.2 \\
Gum bleeding & 4 & 2.4 \\
Epistaxis & 6 & 3.6 \\
Hematemesis & 3 & 1.8 \\
Melena & 13 & 7.8 \\
Menorrhagia & 1 & 0.6
\end{tabular}

Table IV: Distribution of respondents by signs

\section{Signs}

Abdominal tenderness

Coated tongue

Strawberry tongue

Skin rash

Palpable liver

Palpable spleen

Pleural

effusion\&Ascites

Torniquet test

\section{Frequency}

48

20

1

22

41

5

8

6
Percent

28.7

12.0

0.6

13.2

24.6

3.0

4.8

3.6 


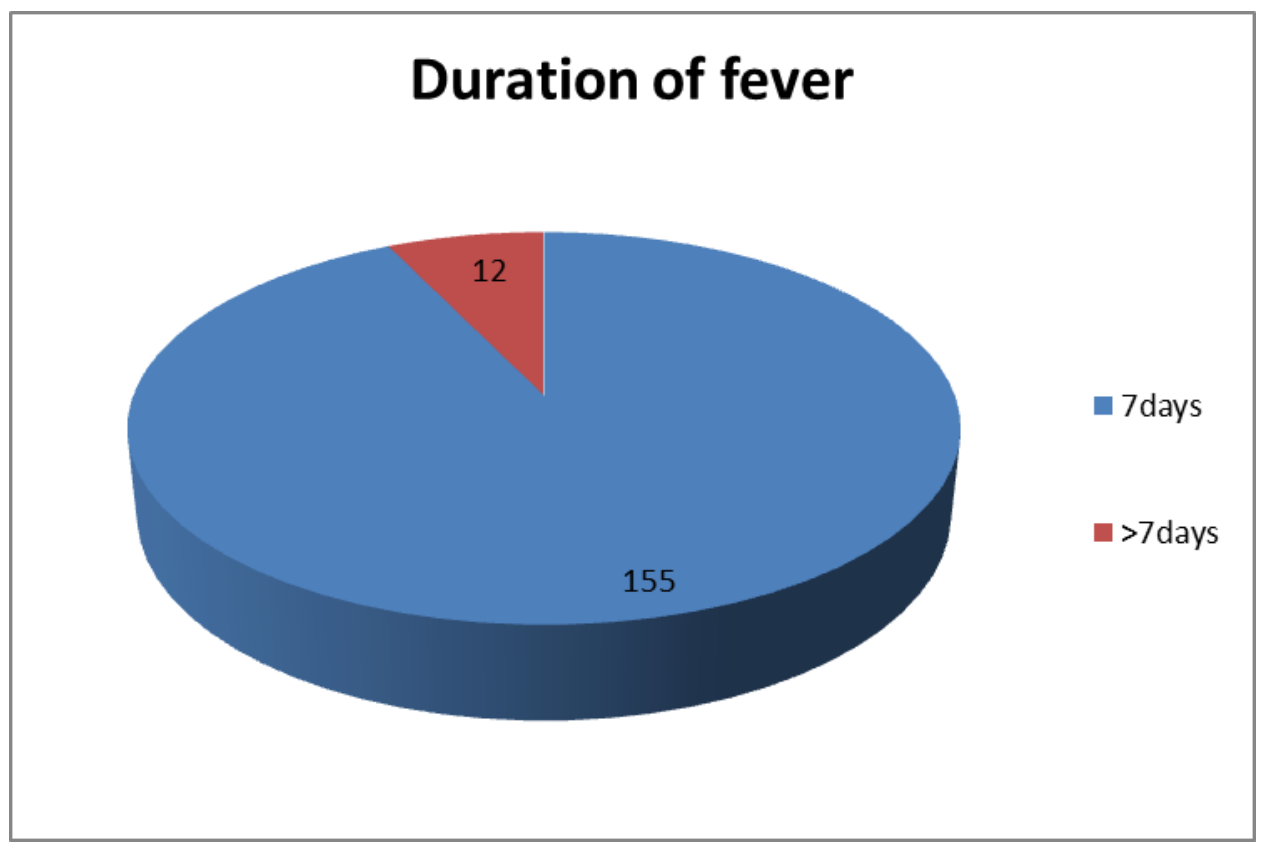

Figure III: Distribution of the respondents by the duration of fever.

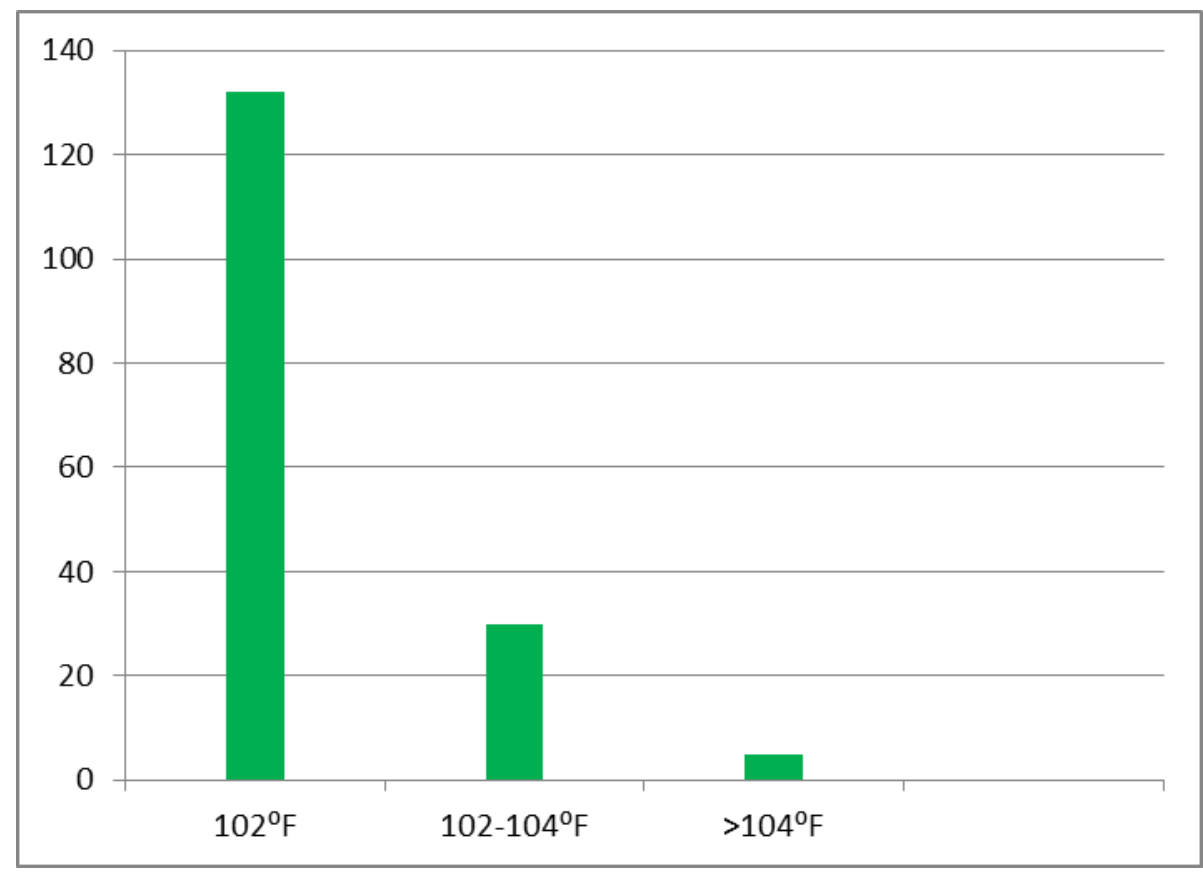

Figure IV: Distribution of the respondents by temperature 
Table V: Distribution of the respondents by associated symptoms

Associated symptoms

Cough

Diarrhea

Cough \& diarrhea

Runny nose

Red-eye

Burning micturation

Mixed symptoms

None

Total

\section{Frequency}

19

13

2

2

4

1

3

123

167
Percent

11.4

7.6

1.2

1.2

2.4

0.6

1.8

73.7

100.0

Table VI: Distribution of the respondents by hematological parameters

WBC count(per cu.mm) Platelet count(per cu.mm) Haematocrit (\%)

$<2000$
$1(0.6)$
$2000-4000$
$16(9.6)$
$4000-11000$
$137(82)$
$>11000$
$13(7.8)$

$$
\begin{gathered}
<100,000 \\
28(16.8) \\
100,000-150,000 \\
36(21.6) \\
150,000-350,000 \\
99(59.3) \\
>350,000
\end{gathered}
$$$$
<30.0
$$$$
\text { 29(17.4) }
$$$$
30.0-35.0
$$$$
85(50.9)
$$$$
35.0-45.0
$$$$
52931.1)
$$$$
>45.0
$$$$
1(0.6)
$$

*Figure in the parenthesis indicates percentage

Table VII: Distribution of patients by status of NS1 antigen

NS1

Positive

Negative

Not done

Total
Frequency

$$
148
$$

5

14

167
Percentage

88.6

3.0

8.4

100.0 
Table VIII: Distribution of patients by Anti-dengue antibody reactivity

$\begin{array}{lcc}\text { Anti-dengue antibody } & \text { Frequency } & \text { Percentage } \\ \text { IgM positive } & 10 & 6.0 \\ \text { IgG positive } & 7 & 4.2 \\ \text { Both IgM \& IgG positive } & 7 & 4.2 \\ \text { Both IgM \& IgG negative } & 57 & 34.1 \\ \text { Not done } & 86 & 51.5 \\ \text { Total } & 167 & 100.0\end{array}$

Table IX: NS1 antigen and anti-dengue antibody status of the patients

NS1

Anti-dengue antibody

Total P-value

IgM(+ve) IgG(+ve) IgM\&IgG IgM\&IgG (- Not done (+ve) ve)

\begin{tabular}{llllllll} 
Positive & $4(2.7)$ & $2(1.4)$ & $4(2.7)$ & $53(35.8)$ & $85(57.4)$ & $148(100.0)$ & \\
& $(40.0)$ & $(28.5)$ & $(57.1)$ & $(92.9)$ & $(98.8)$ & $(88.6)$ & \\
Negative & $1(20.0)$ & $2(40.0)$ & $1(20.0)$ & 00 & $1(20.0)$ & $5(100.0)$ & 0.000 \\
& $(10.0)$ & $(28.6)$ & $(14.3)$ & & $(1.2)$ & $(2.9)$ & \\
\multirow{2}{*}{ Not done } & $5(35.7)$ & $3(21.4)$ & $2(14.9)$ & $4(28.6)$ & 00 & $14(100.0)$ \\
& $(50.0)$ & $(42.9)$ & $(28.6)$ & $(7.0)$ & & $(8.3)$ \\
Total & $10(5.9)$ & $7(4.1)$ & $7(4.1)$ & $57(34.1)$ & $86(51.5)$ & $167(100.0)$ \\
& $(100.0)$ & $(100.0)$ & $(100.0)$ & $(100.0)$ & $(100.0)$ & $(100.0)$
\end{tabular}

Table X: Distribution of the respondents by diagnosis

\section{Diagnosis}

Dengue fever

DF with warning sign

DHF

DHF with shock

Other than dengue

Total

\section{Frequency}

87

51

19

3

7

167

\section{Percent}

52.1

30.5

11.4

1.8

4.2

100.0

\section{Discussion}

There were 99 (59.3\%) boys, and 68 (40.7\%) girls, and the boy-girl ratio was $1.45: 1$ in our study, which is similar to the finding (1.4:1) of Hoque $\mathrm{MS}^{11}$ et al. Commonest age group in our study was

5 - 10 years. Hoque MS et al., Vikram $\mathrm{B}^{12}$ et al., and Haridharshan $\mathrm{G} \mathrm{J}^{13}$ et al. showed similar findings with the common age group being $5-10$ years. Rasul $\mathrm{CH}^{14}$ et al. showed $10-14$ years as the most commonly affected age group. History of fever was present in all cases $(100.0 \%)$. In the 
majority $(92.8 \%)$ of cases, fever persisted for less than seven days. Vomiting was observed in $52.1 \%$ of cases in our study. Hoque $\mathrm{M} \mathrm{S}^{11}$ et al. found vomiting in $56.8 \%$ of cases, and Wang $\mathrm{C} \mathrm{C}^{15}$ et al. found vomiting $(60.5 \%)$ in their study, which is higher in proportion to our finding. In our study, abdominal pain and skin rash was present in $38.3 \%$ and $13.2 \%$ cases respectively, which has similarity with the findings $(35.4 \%)$ and $(15.6 \%)$ of Patel M I ${ }^{16}$ et al. In our study headache was present in $39(23.4 \%)$ cases where as Oncog A $S^{17}$ et al found $11.67 \%$, Patel M I et al ${ }^{16}$ found $74.4 \%$, Shultana $\mathrm{K}^{18}$ found $12.35 \%$ and Hoque $\mathrm{M} \mathrm{S}^{11}$ found $10.0 \%$ cases. There is a wide variation of perception of headache between the studies in children. In our study, $13 \quad(7.8 \%)$ children presented with melena which coincides with the finding $(9.6 \%)$ of Patel $\mathrm{M} \mathrm{I}^{16}$ et al. Only one $(0.6 \%)$ girl presented with menorrhagia. The abdominal tenderness and tourniquet test was positive in $48(28.7 \%)$ and $6(3.6 \%)$ cases, respectively. Shultana $\mathrm{K}^{18}$ found abdominal tenderness in $17.9 \%$ of cases, and the tourniquet test became positive in $4.49 \%$ of cases. In another study in Bangladesh ${ }^{19}$ tourniquet test was positive in $32.0 \%$ of cases. The low proportion of positive tourniquet tests in Indian studies may be due to the darker skin color in Indian children ${ }^{20}$. But in a study in Brazil $^{21}$ tourniquet test was positive in $81.8 \%$ of cases. The liver was found to be palpable in $41(24.6 \%)$ cases in our study, which is similar to the findings of Patel $\mathrm{M} \mathrm{I}^{16}$ et al. (29.3\%) and Hammond $^{22}$ et al. (22.0\%). Pleural effusion and ascites were seen in $8(4.8 \%)$ cases in our study, whereas Chowdhury $\mathrm{J}^{23}$ et al. found pleural effusion and ascites in $25.0 \%$ of cases. Leucopenia was seen in 17 (10.2\%) cases in our study. Patel M $\mathrm{I}^{16}$ et al. and Mishra $\mathrm{S}^{20}$ et al. found leucopenia in $25.77 \%$ and $64.3 \%$, respectively. In our study, thrombocytopenia was seen in $64(38.3 \%)$ cases, and Priyanka ${ }^{24}$ et al. found thrombocytopenia in $59.0 \%$ of cases. It was found that $52.1 \%$ of children suffered from dengue fever (DF), 30.5\% of children from DF with warning signs, $11.4 \%$ of patients from DHF, and DSS $3(1.8 \%)$ in our study. Alam $\mathrm{ABM}^{25}$ et al. found $\mathrm{DF} 40.7 \%$ and DHF $27.8 \%$ cases in their study. Jasmin $\mathrm{RO}^{26}$ et al. reported that, in India, $90.0 \%$ of cases were DF,
$10.0 \%$ were DHF, and no case of DSS. Rahman ${ }^{27}$ et al. found DF $56.0 \%$, DHF $27.6 \%$, and DSS $1.5 \%$ which has a similarity with that of our study. Tewari $\mathrm{K}^{28}$ et al. found $\mathrm{DF} 85.8 \%$, DF with warning sign $11.0 \%$, severe dengue with hemorrhage $2.0 \%$, and severe dengue with severe plasma leakage $1.2 \%$. In our study, $6.0 \%$ cases became IgM positive, $4.2 \%$ cases were $\mathrm{IgG}$ positive, $4.2 \%$ cases became both $\operatorname{IgM}$ and $\mathrm{IgG}$ positive, $34.1 \%$ cases were both $\operatorname{IgM}$ and IgG negative, and anti-dengue antibody test not done in $86(51.5 \%)$ cases. The study showed that $88.6 \%$ of cases were NS1 antigen positive. Among them, $2.7 \%$ of patients became anti-dengue IgM positive, $1.4 \%$ cases were $\mathrm{IgG}$ positive, $2.7 \%$ cases became both $\operatorname{IgM} \&$ IgG positive, $35.8 \%$ patients were both IgM \& IgG negative and antibody test could not be done in $57.4 \%$ cases. An Anti-dengue antibody test was not done in $51.5 \%$ of cases. Among them, most of the cases $(98.8 \%)$ were NS1 positive, and $1.2 \%$ cases were NS1 negative $(P<0.000)$ in our study, which is statistically significant. Mishra $\mathrm{S}^{20}$ et al. found that $47.4 \%$ of cases were NS1 positive, $32.9 \%$ patients became IgM positive, $3.0 \%$ cases were IgG positive, $11.3 \%$ patients became both $\operatorname{IgM}$ and $\mathrm{IgG}$ positive, and $5.1 \%$ cases were NS1 and IgM positive. Three (1.7\%) patients died due to dengue in our study. Among them, two patients died due to DSS, and one patient due to expanded dengue syndrome. Rahman $\mathrm{M}^{27}$ et al. found a case fatality of $1.14 \%$, which is similar to our study.

\section{Conclusion}

Dengue is a self-limiting disease and sometimes becomes devastating if appropriate measurement is not taken timely. A high index of suspicion on the basis of clinical and laboratory parameters help early diagnosis of dengue at their initial stages and thus facilitate monitoring, prompt fluid management, and supportive treatment as per WHO guideline can reduce mortality in patients of severe dengue.

\section{References}

1. Simmons CP, Farrar $\mathrm{J} J$, Nguyen V V, Willis B. Dengue. N Engl J Med 2012;366:1423-32

2. Mustafa MS, Rasotgi V, Jain S, Gupta V.Discovery of fifth serotype of dengue virus(DENV-5): A new 
public dilemma in dengue control. Medical Journal Armed Forces India 2015;71(1):67-70

3. Shirin T, Muraduzzaman AK, Alam AN, Sultana S, Siddiqua $\mathrm{M}$, Khan $\mathrm{MH}$ et al. Largest dengue outbreak of the decade with high fatality may be due to reemergence of DEN-3 serotype in Dhaka, Bangladesh, necessitating immediate public health attention. New microbes and new infections.2019;29:100511

4. Shah MY, Naqash MM, Goel RK, Galhan D.Clinical profile of dengue fever infection in patients admitted in tertiary care centre, Haryana, India. Int $\mathrm{J}$ of Res M Scl 2016;4(6):2146-149

5. Guzman MG, Halstead SB, Artsob H, Buchy $P$, Farrar J, Gubler DJ et al. Dengue: a continuing global threat. Nat Rev Microbiol 2010;8(12 supp) S7-16

6. Srinivasa K,Ajay J,Manjunath GA.Clinical profile and outcome of dengue among hospitalized children-a single centre prospective study.J Pediatr Res 2017;4(02):145-150

7. hhtp://www.who.int/mediacentre/factsheets/fs117/en/

8. Rao M, Aparna A, Jyothi RC.Clinical profile and outcome of dengue infections in children.IOSR 2016;15(2):07-13

9. Trung DT, Thao Le TT, Dung NM et al.Clinical features of dengue in a large Vietnamese cohort: intrinsically lower platelet counts and greater risk for bleeding in adults than children.PLoS Negl Trop Dis 2012;(6):e1679

10. Update on Dengue Management. Bangladesh Society of Medicine 2019.

11. Md.Shafiqul Hoque, Probir KS, ASM Nawshad UA.Clinical profile and outcome of dengue in children admitted in paediatric Intensive care unit in Dhaka Shishu (Children) Hospital, Dhaka, Bangladesh.International Journal of Medicine and Health Research.December 2019;5(12):97-101

12. Vikram B, Jeedan $H$, Virender $K$, Sandeep $K$, Viswas C.Clinical profile and outcome of critically sick patients of dengue admitted in PICU of a tertiary care center. Journal of Pediatric Critical Care 2016;3:20-24

13. Haridharshan GJ, Vjay KK, Gautam K. A Retrospective study on clinical profile and outcome of dengue fever admitted in a tertiary care center. Journal of Pediatric Critical Care 2017;4:23-27

14. Rasul $\mathrm{CH}$, Ahasan HAMN,Rashid AKM M,Khan $\mathrm{MRH}$. Epidemiological factors of Dengue Hemorrhagic Fever in Bangladesh. Indian Pediatr 2002;39:369-72
15. Wang CC, Lee IK, Su MC, Lin HI, Huang YC, Liu SF. Difference in clinical and laboratory characteristics and disease severity between children and adults with dengue virus infection in Taiwan,2002.Trans R Soc Trop Med Hyg 2009;103(9):871-77

16. Patel MI, Patel A. Study of haematological, biochemical profile and clinical presentation in dengue positive patients:82 cases.International Journal of Research in Medical Sciences.2018;6(6):2099-2105

17. Oncog AS, Pondoc J. Clinical and Demographic profile of pediatric patients with dengue fever admitted in three hospital in Tagbilaran city. Pediatr Ther 2017;7(3):1-6

18. Shultana K, Rahman AZMM, M, Baki A Al. I.Dengue Infection in Children: Clinical Profile and Outcome in Dhaka City.American Journal of Pediatrics 2019;5(3):111-115

19. Gomber S, Ramchandran VG, Kumar S, Agarwal $\mathrm{KN}$, Gupta P. Hematological observations as diagnostic markers in dengue hemorrhagic fever-a reappraisal.Indian Paediatr 2001;38:477-81

20 Shubhankar Mishra, Ramya Ramanathan, Sunil Kumar Agarwalla, "Clinical Profile of Dengue Fever in Children: A Study from Southern Odisha, India", Scientifica, vol. 2016, Article ID 6391594, 2016. https://doi.org/10.1155/2016/6391594

21. Srinivasa S,Tanveer N,Chaithanya CN.Clinical profile and ultrasonographic findings in children with dengue fever. Curr Pediatr Res 2014;18(2):87-90

22. Hammond SN, Balmaseda A, Perez L, Tellez Y, Sabrorio SI. Difference in dengue severity in infants, children and adults in a 3-years hospitalbased study in Nicaragua. Am J Trop Med Hyg 2005;73:1063-1070

23. Chowdhury J, Mohanty D et al. Clinical profile and outcome of dengue fever and dengue haemorrhagic fever in pediatric age group. International Journal of Contemporary Pediatrics 2016; 3(2):442-444

24. Patel P, Patel S, Sabalpara M, Shah C, Shah N. Study of hematological and biochemical changes in dengue fever at tertiary care hospital at Ahmedabad. Int J Med Sci Public Heal.2016;5(1)

25. Alam ABM, Sadat S, Swapan Z, Ahmed A. Clinical profile of dengue fever in children. $\mathrm{BJCH}$ 2009;33(2):55-58

26. Jasmin RO,Bhavesh RK,Umed VP,Kshama D,Gajera DV,Thakra ADJ.Clinico-hematological profile and outcome of dengue fever cases 
admitted in 2014 at tertiary care hospital, Rajkot, Gujarat. Natl J Community Med 2017;8(6):320-23

27. Raman MH,Alam AY,Rahman AM,Khan MS,Shapla NR,Aleem MA. Presentation, Management and Outcome of Dengue Fever -A Study of 200 Cases. J Medicine 2013 Apr 12;14(1):18-22
28. Tewari K, Tewari V V, Mehta R. Clinical and Hematological Profile of Patients with Dengue Fever at Tertiary Care Hospital-An observational Study. Mediaterr J Hematol Infect Dis 2018;10(1):e2018021
All correspondence to Dr. Md. Luthfor Rahman, Assistant Professor (Paediatrics), OSD DGHS Mohakhali,Dhaka. Attached to Naogaon Medical College Email:Iuthfor971@yahoo.com 